\title{
Effects of Rhythmic Auditory Stimulation (RAS) on Gait Ability and Symmetry after Stroke
}

\author{
Soon Hyun Lee, PT, MSc'1), Kyoung Jin Lee, PT, MSc ${ }^{1)}$, Chang Ho Song, PT, PhD ${ }^{1)}$ \\ 1) Department of Physical Therapy, Sahmyook University: 26-21, Gongneung 2-dong, Nowon-gu, \\ Seoul, 139-742, Republic of Korea. TEL: +82 2-3399-1630, FAX: +82 2-3399-1638, \\ E-mail: chsong@syu.ac.kr
}

\begin{abstract}
Purpose] The purpose of this study was to investigate the effect of the application of Rhythmic Auditory Stimulation (RAS) to the rehabilitation of stroke patients and its effect on gait symmetry. [Subjects] Twenty-five subjects with stroke performed walking under five conditions and the study progressed following a randomized single-blind cross-sectional design. [Methods] We evaluated the effects of a 5-minute session of RAS. Subjects walked under five conditions: (1) a comfortable speed without external rhythm; (2) non-paretic leg footfall with RAS matching the baseline speed; (3) non-paretic leg footfall with RAS 30\% slower than the baseline speed; (4) paretic leg footfall with RAS beat frequency matching the baseline speed; (5) paretic leg footfall with RAS 30\% faster than the baseline speed. The step time (paretic leg and non-paretic leg), velocity and cadence were measured before and after gait training with RAS [Results] Gait symmetry improved in conditions 4 and 5, but not in conditions 2 and 3. Gait ability was assessed with velocity and cadence. Velocity and cadence improved in conditions 4 and 5. [Conclusion] The results of the study demonstrate the ability of RAS to improve gait symmetry. Thus, RAS should be an effective method for the improvement of the gait symmetry of chronic stroke patients. Key words: Rhythmic Auditory Stimulation (RAS), Gait, Stroke
\end{abstract}

(This article was submitted Oct. 7, 2011, and was accepted Nov. 16, 2011)

\section{INTRODUCTION}

Stroke is caused by blocked blood flow to the brain $\mathbb{1}$ and it causes motor function disorder.

Hemiplegia can be the main cause of disorder and disability and it arises from functional limitations in muscle control which limit transfer and movemente. Hemiplegic patients show a variety of disorders such as paralysis, muscle weakness, spasticity, abnormal muscle contractions, reduced balance ability, and reduced gait ability $\$$.

In particular, the reduced gait ability can degrade the quality of life and gait recovery is one of the most important purposes in the rehabilitation of stroke patients 5 . The cause of reduced gait ability is gait asymmetry which is caused by diminished stance time, narrow step length, and asymmetrical stride length 9 . Recent studies using rhythmic auditory stimulation(RAS) have reported improvements in the asymmetry of gait and the gait ability in various ways 10 . The study using RAS was started by study for Parkinson and hemiplegic patients $10-13$. Studies of hemiplegic patients have been actively conducted using various stimuli and environments 14 . The gait training by Schauer and Mauritz used music improved gait symmetry. The gait training by Ford 10 focused on the tempo on a treadmill and it improved the movements of the upper and lower extremities. Roerdink

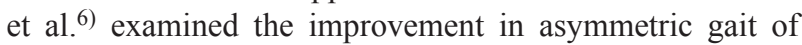
acoustically paced walking by stroke patients following a metronome in three-speed treadmill gait training. Gait asymmetry was reduced as well as the recovery ability from perturbation due to abrupt change in the speed of treadmill. Thus, the effectiveness of RAS at improving the recovery from perturbation and gait symmetry was demonstrated. In addition, Thaut et al. 10 reported in their studies that a group following a metronome and music showed improved gait velocity and symmetry compared to a NDT / Bobath group demonstrating RAS is effective at improving the gait of hemiplegic patients.

RAS has been performed for gait focusing on tempo until now, but it did not specifically suggest the applications and most studies have been performed in the restricted environment of a treadmill. Thus, this study investigated the effect of RAS on the improvement of gait ability and gait symmetry between the paretic leg and non-paretic leg in gait performed on level ground, rather than in the restricted environment of a treadmill.

\section{SUBJECTS AND METHODS}

The subjects of this study were chronic stroke patients in a hospital, who had been hospitalized for 6 months to 2 years and who could perform gait independently for a minimum 5 minutes. Twenty-five patients were recruited from among 28 patients. Two patients, who were incapable of independent gait, and 1 patient who refused to participate to the experiment were excluded. Five gait trials, each with a total walking time of 5 minutes, were performed under the 
following conditions and in the following order: (1) at an individually chosen comfortable speed without RAS; (2) non-paretic leg footfall with RAS matching the baseline speed; (3) non-paretic leg footfall with RAS 30\% slower than the baseline speed; (4) paretic leg footfall with RAS beat frequency matching the baseline speed; and (5) paretic leg footfall with RAS 30\% faster than the baseline speed. In a preliminary experiment conducted at different speeds of plus or minus $10 \%, 20 \%, 30 \%, 40 \%$ and $50 \%$ of baseline speed, auditory stimulation below $30 \%$ did not affect gait variables and subjects found it hard to carry out the experiment above $30 \%$. This led us to set the speeds of $\pm 30 \%$ of baseline speed.

RAS was provided by a metronome (TU-88, BOSS, China) adjusted to each subject's step time $(\mathrm{m} / \mathrm{s})$, which was measured by a gait analysis system before training. Gait training with RAS was performed in an independent space for 5 minutes each time and the time for training consisted of 8 minutes in total (warm-up for 3 minutes and gait training for 5 minutes). Subjects performed warm-up before gait in time with the beat by marking time while listening to acoustic cues in a standing or sitting position. Warm-up was performed to increase the adaptation to RAS and tension the muscles. There was an interval of 10 minutes between the trials to eliminate any remaining effects which could have influenced the next trial. Gait training focused on the timing of the contact of the feet on the ground and the patients were asked to perform heel strike in time with RAS. In the paretic leg footfall with RAS, the patients walked, stepping the paretic leg on the ground in time with the sound from the metronome, and in the non-paretic leg footfall with RAS, the patients walked, stepping the non-paretic leg on the ground in time with the sound from the metronome. To prevent reduced concentration because of external interference and noise, Bluetooth Wireless Headphones (MDR-RF4000 $\mathrm{K}$, Sony, Japan; effective distance: $30 \mathrm{~m}$ ) were used and stopwatches (HS-80W-1D, Casio, Japan) measured the gait time of 5 minutes. To collect data for quantitative gait analysis, a gait analyzer (GAITRite, CIR system Inc, USA, 2008) was used to measure the temporal and spatial gait ability. The reliability of the tester was $r=0.09$, and the correlation coefficient (ICC $=0.99$ ) in all gait measurements of comfortable gait velocity was above $0.96{ }^{17}$. For all subjects, the test was conducted before and after the training, and step time, gait velocity and cadence were averaged from three repeated measurements. To minimize muscle fatigue, 1 minute of rest time was inserted between the measurements and the tests were conducted without a gait assistance device. The data collected by the software of the gait analyzer was output as Excel files and processed using the Excel v2007 program, then the symmetry of gait was calculated as described below. For calculating accurate symmetry, among various formulae suggested in the preceding studies, the formula appropriate for this study was selected.

The symmetry index (SI) is the ratio of the sum of non-paretic side value and the paretic side value and difference between non-paretic side value and the paretic side value ${ }^{18}$. The gait asymmetry (GA) was calculated by multiplying the log value of the paretic side value/ non-paretic side value by 100 19. The symmetry ratio (SR) was obtained
Table 1. Subject characteristics

\begin{tabular}{lc}
\hline & Subjects \\
\hline Gender (male/female) & $18(14 / 11)$ \\
Age (years) & $64.31 \pm 8.2$ \\
Height $(\mathrm{cm})$ & $164.1 \pm 8.4$ \\
Weight $(\mathrm{kg})$ & $62.20 \pm 9.3$ \\
Type of stroke: hemorrhage/infarction & $12 / 13$ \\
Hemiparetic side: right/left & $13 / 12$ \\
Brunnstrom stage of paretic arm stage & $\mathrm{I} / \mathrm{II} / \mathrm{III} / 8 / 12 / 5$ \\
Duration (months) & $12.8 \pm 7.5$ \\
\hline
\end{tabular}

Note. Values are expressed as mean \pm standard deviation (SD).

by dividing the paretic side value by the non-paretic side value 20. In this study, both sides' step times were used for calculating the symmetry. To confirm the normality of variables, the Shapiro-Wilk normality test was conducted. Repeated measures ANOVA was used to identify the differences between conditions and the Bonferroni test was used for post hoc tests. All analyses were performed using SPSS 18.0 (SPSS Inc., San Rafael, CA, USA) with a significance level of less than 0.05 .

\section{RESULTS}

The general characteristics of the 21 subjects are shown in Table 1.

The changes in symmetry according to the conditions of gait training with / without RAS are shown in Table 2. For all of the symmetry variables, there were some significant differences between the RAS conditions and baseline after gait training with RAS $(\mathrm{p}<0.05)$.

For SI, there were no significant differences between condition 1 , and conditions 2 and 3 , but conditions 4 and 5 showed significant decreases $(\mathrm{p}<0.05)$ from condition 1 , indicating that paretic leg footfall with RAS improved gait symmetry. The decrease of condition 5 was even more significant than that of condition $4(\mathrm{p}<0.05)$. For $\mathrm{GA}$, there was no significant difference between conditions 1 and 2, but conditions 3, 4 and 5 showed significant decreases $(p<0.05)$ from condition 1. Conditions 4 and 5 showed more significant improvements than condition $3(\mathrm{p}<0.05)$ indicating that paretic leg footfall with RAS realizes more significant improvements than non-paretic leg footfall with RAS. In particular, there was an even more significant decrease in condition $5(\mathrm{p}<0.05)$. For SR, there were no significant differences between condition 1 , and conditions 2 and 3 , but conditions 4 and 5 showed significant decreases from condition $1(\mathrm{p}<0.05)$ indicating that paretic leg footfall with RAS realizes more significant improvement than non-paretic leg footfall with RAS. Condition 5 showed a more significant decrease than condition $4(\mathrm{p}<0.05)$.

The changes in gait ability according to the conditions of gait training with / without RAS are shown in Table 3. Gait velocity and cadence showed significant differences frome the baseline (condition 1) after gait training with RAS ( $p<0.05)$. Conditions 4 and 5 showed more significant improvements than the other conditions $(\mathrm{p}<0.05)$ indicating 
Table 2. The changes in symmetry according to the conditions

\begin{tabular}{lcccccc}
\hline & $\mathrm{C} 1$ & $\mathrm{C} 2$ & $\mathrm{C} 3$ & $\mathrm{C} 4$ & $\mathrm{C} 5$ & $\mathrm{~F}$ \\
\hline SI & $30.28 \pm 22.01$ & $22.79 \pm 14.57$ & $21.43 \pm 13.51$ & $19.98 \pm 12.66$ & $18.45 \pm 12.08$ & $3.694^{*}$ \\
GA & $19.33 \pm 10.57$ & $16.20 \pm 9.34$ & $14.77 \pm 8.70$ & $13.47 \pm 8.42$ & $12.57 \pm 8.52$ & $9.787^{*}$ \\
SR & $1.21 \pm 0.14$ & $1.15 \pm 0.14$ & $1.13 \pm 0.15$ & $1.12 \pm 0.14$ & $1.09 \pm 0.15$ & $23.952^{*}$ \\
\hline
\end{tabular}

Note. Values are expressed as mean SD. ${ }^{*}$, significant difference from the baseline. SI: Symmetry Index. GA: Gait Asymmetry. SR: Symmetry Ratio. C1: comfortable speed without RAS. C2: non-paretic leg footfall with RAS matching the baseline speed. C3: non-paretic leg footfall with RAS 30\% slower than the baseline speed. C4: paretic leg footfall with RAS matching the baseline speed. C5: paretic leg footfall with RAS 30\% faster than the baseline speed.

Table 3. The changes in gait abilities according to the conditions

\begin{tabular}{ccccccc}
\hline & C1 & C2 & C3 & C4 & \multicolumn{1}{c}{ C5 } & \multicolumn{1}{c}{ F } \\
\hline Velocity & $69.68 \pm 19.84$ & $65.36 \pm 20.54$ & $63.39 \pm 21.52$ & $76.90 \pm 19.42$ & $81.53 \pm 18.63$ & $25.205^{*}$ \\
Cadence & $102.15 \pm 16.14$ & $98.71 \pm 16.43$ & $97.50 \pm 19.06$ & $110.57 \pm 18.07$ & $114.77 \pm 20.22$ & $144.647^{*}$ \\
\hline
\end{tabular}

Note. Values are expressed as mean SD. *, significant difference from the baseline. C1: comfortable speed without RAS. C2: non-paretic leg footfall with RAS matching the baseline speed. C3: non-paretic leg footfall with RAS 30\% slower than the baseline speed. C4: paretic leg footfall with RAS matching the baseline speed. C5: paretic leg footfall with RAS 30\% faster than the baseline speed.

that paretic leg footfall with RAS improved velocity and cadence. In particular, condition 5 showed an even more significant decrease than condition $4(\mathrm{p}<0.05)$.

\section{DISCUSSION}

The present study showed results similar to normal gait after gait training with RAS, because the gait training using the acoustic cue improved asymmetric gait of stroke patients and it had a positive effect on the gait velocity. This study investigated the effect of gait training with RAS on the gait asymmetry of the paretic and non-paretic sides, and gait ability improvement; in order to devise a more effective way of gait training with RAS.

Paretic leg footfall with RAS matching the baseline speed \& $30 \%$ faster speed realized significant improvements in symmetry and gait ability, but in non-paretic leg footfall with RAS, there was no significant effect. Thus, we demonstrated that paretic leg footfall with RAS is an effective symmetry and gait ability of hemiplegic patients.

In paretic leg footfall with RAS matching the baseline speed and 30\% faster, both symmetry and gait ability improved significantly. It implying that cue control of the paretic leg footfall at baseline speed and 30\% faster shorten the slow step time of the paretic leg, improving the step time on the paretic side positively affecting gait. In a study that classified the gait cycle into 'stance phases' and 'swing phases', the gait cycle was improved by gait training with RAS . Hausdorff et al.13 reported that gait training with RAS enhanced the stance phase of patients with Parkinson's disease. Another study reported weight shift ability improvement resulted in improved swing phase time and walking 11 . Roerdink et al. concluded that training with RAS would reduce the step time of stroke patients. The results of our present study are in agreement with these previous reports.

When normal speed and 30\% slower speed with RAS were used for the non-paretic leg, there were no significant effects on symmetry or gait ability. However, on GA, one of the items of symmetry in non-paretic leg footfall with RAS matching a speed $30 \%$ faster than the baseline speed, there was a significant effect. The results of the present study demonstrate the improvement of gait symmetry and gait ability by gait training with RAS for hemiplegic patient. Many preceding studies have reported that gait training with RAS improved gait ability and symmetry. Thaut et al. ${ }^{14}$ reported that the use of RAS improved gait velocity and stride length of stroke patient, and Schauer and Mauritz reported that it improved gait velocity $(\mathrm{P}=0.008)$ and stride length $(P=0.009)$ in their experiments involving stroke patients. Del Olmo and Cudeiro and Rochester et al.2reported that gait training with RAS for Parkinson disease patients improved gait velocity, stride length, and cadence demonstrating its effect on gait ability.

The shortened step length of the paretic side, due to impaired propulsion generating capacity, makes gait velocity slow and reduces gait ability ${ }^{23}$. This impaired propulsion is caused by the weakened muscle strength of the triceps surae and the loss of muscle contraction timing, and recovery of this function would improve gait ability ${ }^{2}$. In addition, Roerdink and Beek 15 reported that improved trunk rotation at the time of gait, improved the propulsion at the time of the stance on the paretic side. The implementation of RAS using metronome beats improved the pelvis and trunk movement (i.e the trunk rotation ability) $\sqrt{10}$ and significant changes in EMG muscle activity of the gastrocnemius were seen after 3 weeks of RAS training ${ }^{14}$.

The present study showed that gait training with RAS for hemiplegic patients is effective at improving gait symmetry and gait ability. The results suggest rehabilitation and therapeutic interventions for improving hemiplegic patient's gait symmetry and gait ability. We think further studies seeking the most effective use of RAS and verification of the long-term effects of gait training with RAS are needed. 


\section{ACKNOWLEDGMENT}

This research was supported by a Sahmyook University Research Grant.

\section{REFERENCES}

1) Feigin VL: Stroke epidemiology in the developing world. Lancet, 2005, 365: 2160-2161. [Medline CrossRef

2) Langhorne P, Coupar F, Pollock A: Motor recovery after stroke: a systematic review. Lancet Neurol, 2009, 8: 741-754. [Medline] [CrossRef

3) Arene N, Hidler J: Understanding motor impairment in the paretic lowe limb after a stroke: a review of the literature. Top Stroke Rehabil, 2009, 16 346-356. Medline [CrossRef

4) Franceschini M, Carda S, Agosti M, et al.: Walking after stroke: what does treadmill training with body weight support add to overground gait training in patients early after stroke?: a single-blind, randomized, controlled trial. Stroke, 2009, 40: 3079-3085. [Medline] [CrossRef

5) Bohannon RW, Horton MG, Wikholm JB: Importance of four variables of walking to patients with stroke. Int J Rehabil Res, 1991, 14: 246-250. [Medline [CrossRef

6) Roerdink M, Lamoth CJ, Kwakkel G, et al.: Gait coordination after stroke: benefits of acoustically paced treadmill walking. Phys Ther, 2007, 87: 1009-1022. [Medline CrossRef

7) Roerdink M, Lamoth CJ, van Kordelaar J, et al.: Rhythm perturbations in acoustically paced treadmill walking after stroke. Neurorehabil Neura Repair, 2009, 23: 668-678. Medline CrossRef

8) Hayden R, Clair AA, Johnson G, et al.: The effect of rhythmic auditory stimulation (RAS) on physical therapy outcomes for patients in gait training following stroke: a feasibility study. Int J Neurosci, 2009, 119: 2183 2195. [Medline] CrossRef]

9) Pelton TA, Johannsen L, Chen H, et al.: Hemiparetic stepping to the beat: asymmetric response to metronome phase shift during treadmill gait. Neurorehabil Neural Repair, 2010, 24: 428-434. [Medline] [CrossRef]

10) Ford MP, Wagenaar RC, Newell KM: The effects of auditory rhythms and instruction on walking patterns in individuals post stroke. Gait Posture, 2007, 26: 150-155. Medline CrossRef

11) del Olmo MF, Arias P, Furio MC, et al: Evaluation of the effect of training using auditory stimulation on rhythmic movement in Parkinsonian patients-a combined motor and [18F]-FDG PET study. Parkinsonism Relat Disord, 2006, 12: 155-164. Medline] [CrossRef]

12) Willems AM, Nieuwboer A, Chavret F, et al.: The use of rhythmic auditory cues to influence gait in patients with Parkinson's disease, the differentia effect for freezers and non-freezers, an explorative study. Disabil Rehabil, 2006, 28: 721-728. [Medline] CrossRef

13) Hausdorff JM, Lowenthal J, Herman T, et al.: Rhythmic auditory stimulation modulates gait variability in Parkinson's disease. Eur J Neurosci, 2007, 26: 2369-2375. [Medline] [CrossRef]

14) Thaut MH, McIntosh GC, Rice RR: Rhythmic facilitation of gait training in hemiparetic stroke rehabilitation. J Neurol Sci, 1997, 151: 207-212. Medline [CrossRef

15) Schauer M, Mauritz KH: Musical motor feedback (MMF) in walking hemiparetic stroke patients: randomized trials of gait improvement. Clin Rehabil, 2003, 17: 713-722. [Medline] [CrossRef]

16) Thaut MH, Leins AK, Rice RR, et al.: Rhythmic auditory stimulation improves gait more than NDT/Bobath training in near-ambulatory patients early poststroke: a single-blind, randomized trial. Neurorehabil Neural Repair, 2007, 21: 455-459. [Medline] [CrossRef

17) van Uden CJ, Besser MP: Test-retest reliability of temporal and spatial gait characteristics measured with an instrumented walkway system (GAITRite). BMC Musculoskelet Disord, 2004, 5: 13. [Medline] [CrossRef

18) Patterson KK, Gage WH, Brooks D, et al.: Evaluation of gait symmetry after stroke: a comparison of current methods and recommendations for standardization. Gait Posture, 2010, 31: 241-246. [Medline [CrossRef

19) Plotnik M, Giladi N, Hausdorff JM: A new measure for quantifying the bilateral coordination of human gait: effects of aging and Parkinson's disease. Exp Brain Res, 2007, 181: 561-570. [Medline] [CrossRef]

20) Plotnik M, Giladi N, Balash Y, et al.: Is freezing of gait in Parkinson's disease related to asymmetric motor function? Ann Neurol, 2005, 57: 656663. Medline [CrossRef

21) del Olmo MF, Cudeiro J: Temporal variability of gait in Parkinson disease: effects of a rehabilitation programme based on rhythmic sound cues. Parkinsonism Relat Disord, 2005, 11: 25-33. [Medline] [CrossRef]

22) Rochester $L$, Hetherington $V$, Jones $D$, et al.: The effect of external rhythmic cues (auditory and visual) on walking during a functional task in homes of people with Parkinson's disease. Arch Phys Med Rehabil, 2005, 86: 999-1006. Medline [CrossRef

23) Balasubramanian CK, Bowden MG, Neptune RR, et al.: Relationship between step length asymmetry and walking performance in subjects with chronic hemiparesis. Arch Phys Med Rehabil, 2007, 88: 43-49. Medline CrossRef

24) Turns LJ, Neptune RR, Kautz SA: Relationships between muscle activity and anteroposterior ground reaction forces in hemiparetic walking. Arch Phys Med Rehabil, 2007, 88: 1127-1135. [Medline] [CrossRef]

25) Roerdink M, Beek PJ: Understanding inconsistent step-length asymmetries across hemiplegic stroke patients: impairments and compensatory gait. Neurorehabil Neural Repair, 2011, 25: 253-258. 